\title{
Fontes para a história da ginecologia e obstetrícia no Brasil
}

\author{
Sources for the history of gynecology \\ and obstetrics in Brazil
}

\section{Larissa Velasquez de Souza}

Doutoranda, Programa de Pósgraduação em História das Ciências e da Saúde/Casa de Oswaldo Cruz.

larissasouzavelasquez@gmail.com

Recebido em 3 out. 2017. Aprovado em 23 fev. 2018.
SOUZA, Larissa Velasquez de. Fontes para a história da ginecologia e obstetrícia no Brasil. História, Ciências, Saúde - Manguinhos, Rio de Janeiro, v.25, n.4, out.-dez. 2018, p.1129-1146.

Resumo

Este artigo se insere no debate sobre a medicalização do parto no Brasil, a partir do levantamento de fontes realizado em bases de dados de bibliotecas brasileiras, e apresenta como objetivo principal a disponibilização sistematizada dos títulos das publicações científicas especializadas existentes nas áreas de ginecologia e obstetrícia no século XX, especialmente na primeira metade desse século. Considerando a relevância da análise de periódicos na construção do conhecimento científico no Brasil, assim como a importância das revistas especializadas na constituição e no desenvolvimento de especialidades médicas, apresentou-se um detalhamento desses acervos, considerando localização, período de publicação e local de produção, apresentando mais detidamente o acervo situado na biblioteca da Maternidade-Escola da Universidade Federal do Rio de Janeiro.

Palavras-chave: ginecologia; obstetrícia; periódicos; fontes; medicalização.

\begin{abstract}
This article contributes to the debate about the medicalization of childbirth in Brazil through a literature review of databases of Brazilian libraries. Its main goal is to enable the systematized availability of specialized scientific publications in gynecology and obstetrics in the twentieth century, especially 1900 to 1950. In view of the importance of the analysis of journals in the development of scientific knowledge in Brazil and the importance of specialized journals in constituting and developing medical specializations, details of these archives are presented, including their location, when they were published, and where they were produced, paying particular attention to the archive in the library of the Teaching Maternity Hospital (Maternidade-Escola) of the Universidade Federal do Rio de Janeiro.
\end{abstract}

Keywords: gynecology; obstetrics; journals; sources; medicalization.

http://dx.doi.org/10.1590/S0104-59702018000500011 
$\mathrm{E}$ ste artigo tem como principal objetivo apresentar o levantamento realizado em algumas bases de periódicos do Brasil, com a finalidade de identificar os títulos de publicações científicas existentes na área de ginecologia e obstetrícia, contemplando a primeira metade do século XX, a fim de somar com o conhecimento produzido sobre esses campos, destacando certas temáticas abordadas nessas fontes.

A investigação sobre a medicalização do corpo feminino em diferentes contextos, inserida no processo de medicalização geral da sociedade, desenvolveu-se nas últimas décadas por pesquisadores de diversas áreas do conhecimento. A regulação do corpo feminino por meio de normas, em diferentes sociedades, expandiu-se para a esfera médica na qual o discurso da "natureza feminina" embasava a retórica médico-social com intervenções médicas que ampliaram o processo em questão (Vieira, 2002).

O desenvolvimento da obstetrícia e da ginecologia, no século XIX, instrumentalizou esse percurso. Nesse período, as duas especialidades separaram-se e passaram a constituir dois campos distintos de atuação sobre o corpo feminino. A ideia de medicina da mulher, segundo Fabíola Rohden (2009), estabeleceu-se a partir da ginecologia, ampliando a medicalização do corpo feminino em contraste ao masculino, atuando no cuidado das doenças femininas a partir das diferenças sexuais.

No contexto do desenvolvimento de novos conhecimentos e novas técnicas, as duas especialidades inseriram-se no movimento mais amplo de especialização da medicina, e, com as reformas ocorridas na Faculdade de Medicina do Rio de Janeiro nos anos de 1879, 1881 e 1882, foi criada a cadeira de clínica obstétrica e ginecológica (Rohden, 2009). A construção como campo demandou, segundo Rohden, o investimento dos médicos no "gerenciamento da saúde feminina e da reprodução", além da "normalização das práticas relativas ao corpo feminino", com o objetivo de construir conhecimento sobre esse corpo em torno da diferença sexual e manter uma hierarquia entre os gêneros (p.75-79).

As reformas no campo da medicina demandaram mecanismos de legitimação e sustentação, e para isso contribuiu o desenvolvimento da imprensa médica com a criação de revistas como Brasil Médico, em 1887, ligadas à Faculdade de Medicina do Rio de Janeiro, e Gazeta Médica da Bahia, em 1866 (Rohden, 2009). Os temas da sexualidade e reprodução estavam presentes nesses periódicos generalistas antes de conquistarem espaço próprio nas revistas especializadas, permanecendo nas seções específicas das revistas médicas gerais.

Nesse período, as revistas dedicadas às especialidades da medicina começavam a se propagar e se estabeleceram como espaços de divulgação de novas descobertas para os pares, do desenvolvimento de técnicas e abordagens, além de apresentação da produção exterior sobre a temática em questão, contribuindo para a delimitação das especificidades de diferenciados campos. A vulgarização da ciência, por sua vez, ficava por conta das notícias veiculadas em jornais de ampla circulação (Sá, 2006).

Essas revistas se configuram como importantes fontes para a investigação histórica, tendo seu início marcado pelo contexto das mudanças na prática historiográfica decorrentes da nova história francesa, que propunha "novos objetos, novos problemas e novas abordagens", assim como da renovação do marxismo, com o reconhecimento da importância de elementos culturais, na segunda metade do século XX (Luca, 2008). 
No Brasil, de acordo com Tania de Luca (2008), até a década de 1970 se elaborava a história da imprensa, mas não a história constituída por meio da imprensa, com uma histografia ainda marcada pelo ideal de neutralidade e objetividade que estabelecia uma hierarquia dos documentos. A ampliação das temáticas, incorporando pesquisas sobre o corpo, mulheres, festas, entre outros, indicavam, segundo a autora, o "alargamento do campo" e a "fragmentação da disciplina", alterando a concepção de documento e sua análise (p.113).

Afastando-se de uma concepção do uso instrumental dos periódicos como "meros receptáculos de informações a serem selecionadas, extraídas e utilizadas ao bel prazer do pesquisador" (Luca, 2008, p.116), a análise desse tipo de documento, segundo a autora, deve considerar aspectos das matérias e de conteúdo da publicação, além de autoria, públicoalvo e relações com mercado e publicidade (no que diz respeito à imprensa de ampla divulgação, mas que pode ser incorporado na análise de periódicos científicos quando se consideram as relações com indústria farmacêutica e demais patrocinadores). Luca (2008, p.139) acrescenta ainda que "o conteúdo em si não pode ser dissociado do lugar ocupado pela publicação na história da imprensa", o que pode ser aplicado, no caso da imprensa médica, por suas influências internas e externas e todo o seu trabalho de legitimação dos campos da medicina ginecológica e obstétrica.

Os periódicos especializados em áreas diferenciadas da medicina têm se constituído como fonte amplamente utilizada na análise de processos de formação médica, nas práticas de terapias de análise do desenvolvimento do conhecimento científico, entre outras temáticas. No que diz respeito aos periódicos voltados para as áreas de ginecologia e obstetrícia no Brasil, pode-se observar que foram objetos principais de trabalhos que investigaram o processo histórico de configuração desses campos (Rohden, 2009; Martins, 2004; Mott, 2002). Além disso, contribuíram para pesquisas que trataram da medicalização do corpo feminino por meio de temáticas como o uso de hormônios e sua abordagem em periódicos (Freitas, 2005; 2008; Bonan, Teixeira, Nakano, 2017; Menezes, 2012).

A desmistificação da ideia de neutralidade do discurso científico pela análise histórica de periódicos é apontada por Patricia Freitas (2008) como uma das contribuições necessárias do historiador, cabendo a ele compreender, na análise de periódicos das áreas de ginecologia e obstetrícia, como as experiências do corpo feminino, tais como menstruação, gravidez, parto e menopausa, "foram interpretadas e como essas interpretações chegam até as mulheres em forma de modos de conduta, proibições, censuras que podem ser apresentadas como recomendações, dicas..." (p.36).

Por considerar a relevância do contexto apresentado, entendendo as revistas científicas como espaços importantes de construção, divulgação e legitimação do conhecimento científico e da prática médica, apresenta-se neste espaço o resultado do mapeamento de periódicos científicos das especialidades de ginecologia e obstetrícia publicados no Brasil a partir do século XX.

\section{A construção do campo da ginecologia e obstetrícia}

A produção científica na área da ginecologia e obstetrícia teve, nas revistas especializadas no tema, espaço importante para sua divulgação ao final do século XIX, com expressão 
significativa a partir do início do século XX, acompanhando a trajetória da produção científica geral da medicina em busca de legitimação da comunidade médica e das diversas especialidades.

Essa especialização estava atrelada à ampliação do conhecimento científico sobre a medicina, e os periódicos, nesse contexto, constituíam espaços nos quais os médicos se apresentavam como "cientistas", profissionais de um ramo específico de saber (Sá, 2006). Estava em desenvolvimento a formação de uma "ciência" nacional, processo no qual "os homens de ciência que se formaram e atuaram na virada do século XIX para o XX percebiam-se como os primeiros a contribuírem para a fixação definitiva de instituições, padrões de análise e normas de conduta social para a ciência brasileira" (p.119).

O conhecimento produzido na área da obstetrícia data da segunda metade do século XVIII, período em que se observa uma grande produção de livros sobre o tema. O desenvolvimento dessa especialidade como ciência nas faculdades de medicina europeias ocorreu na primeira metade do século XIX, resultando no processo de institucionalização do saber médico (Martins, 2004).

Assim como as demais especialidades médicas, a consolidação do campo da obstetrícia no Brasil também enfrentou dificuldades em seu desenvolvimento, tendo como principal desafio a ausência de maternidades para o seu ensino prático, já que as primeiras instituições foram criadas no final do século XIX e início do XX (Mott, 2002). A construção do conhecimento na área da medicina da mulher foi desenvolvida, institucionalmente, a partir da atuação de médicos que ministravam aulas nas primeiras faculdades criadas no Brasil em 1835: a Faculdade de Medicina do Rio de Janeiro e a Faculdade de Medicina da Bahia. Dessa forma, médicos de grande reputação atuaram no estabelecimento das áreas de ginecologia e obstetrícia por conta da prática como mestres nessas escolas, divulgando essas especialidades para diversos médicos parteiros e ginecologistas em formação (Martins, 2004).

O interesse desses profissionais pelo parto surgiu desde o século XVI, porém, durante os séculos XVIII e XIX é "que a prática dos cuidados com o corpo feminino, considerada como competência exclusiva dos médicos, irá desenvolver-se, organizar-se, enfim, legitimar-se" (Vieira, 2002, p.23). Esse processo de reconhecimento estabeleceu-se paralelamente ao de outras especialidades médicas no século XIX, com o surgimento de periódicos científicos no Brasil, construídos ao modelo europeu de tradução de livros, verbetes, enciclopédias e artigos de revistas e jornais científicos estrangeiros, organizando-se como espaços de institucionalização, legitimação e divulgação da ciência (Ferreira, 1999; Freitas, set.-dez. 2006). A produção de publicações médicas sobre o corpo feminino buscava explicar a anatomia e a fisiologia desse corpo, prescrever condutas e classificar doenças, indicando os respectivos tratamentos (Martins, 2004).

Na análise do campo em questão, a construção da ginecologia e da obstetrícia no Brasil como disciplinas responsáveis pela construção e reprodução de conhecimento sobre as mulheres fundamentou-se em parâmetros de distinção entre os sexos, com destaque para a função reprodutora da mulher (Freitas, 2005; Martins, 2004). As revistas especializadas nessas áreas reproduziam os feitos e os estudos do campo da ginecologia, como os estudos sobre cirurgias ginecológicas que, de acordo com Freitas, eram realizados a partir da segunda 
metade do século XIX, com debates até o início do século XX, sendo esses identificados em artigos publicados (Freitas, 2005). As especialidades de ginecologia e obstetrícia "conquistaram um espaço exclusivo de produção e divulgação de conhecimentos, exposição de ideias, defesa de posições a respeito de doutrinas e debates de temas polêmicos" (Martins, 2004, p.181).

No Brasil, um levantamento realizado no ano de 2016 identificou 47 títulos de periódicos especializados em ginecologia e/ou obstetrícia, publicados entre 1861 e 2010, disponíveis em acervos abertos ao público. Nesse conjunto, sete títulos de periódicos eram continuação de títulos anteriores, contabilizando, na realidade, quarenta publicações diferentes, conforme demonstrado nas tabelas que acompanham este estudo.

A localização ilustra a produção científica do período na área da ginecologia e obstetrícia, e o destaque de alguns assuntos aponta para os debates e controvérsias do campo, assim como as contribuições das demandas internacionais sobre diversas temáticas presentes em algumas dessas revistas. A construção de uma medicina da mulher e a atuação dos médicos nas recém-criadas maternidades, além do registro da trajetória histórica do uso de medicamentos e técnicas diferenciadas na assistência à saúde sexual e reprodutiva da mulher, apresentam-se como alguns dos temas identificados pelos pesquisadores envolvidos.

\section{Percurso metodológico do levantamento das fontes}

O levantamento em questão foi realizado no âmbito da pesquisa intitulada "Medicalização dos nascimentos no Brasil", desenvolvida pela Casa de Oswaldo Cruz/ Fiocruz, com a participação de pesquisadores nas áreas de história, antropologia, medicina, enfermagem e biblioteconomia, e tem como objetivo principal analisar, em perspectiva histórica e sociológica, o processo de medicalização do parto no Brasil, considerando as técnicas e práticas médicas envolvidas nessa ação, incorporadas no século XX.

O levantamento foi realizado nas seguintes bases de dados: Biblioteca Virtual em Saúde, seção Catálogo de Revistas Científicas; Catálogo Coletivo Nacional - Ibibct; Biblioteca de História das Ciências e da Saúde/COC/Fiocruz-Base de Periódicos; Biblioteca de Ciências Biomédicas - Icict/Fiocruz, seção Periódicos Científicos; Biblioteca da Academia Nacional de Medicina, catálogo on-line; Biblioteca Nacional, seção Catálogo de Periódicos; Bibliotecas da UFRJ - Base Minerva; Bibliotecas da USP - Portal de busca integrada; Unicamp - Sistema de Bibliotecas da Unicamp.

A estratégia de busca desenvolveu-se a partir da utilização de descritores por assunto, a saber: "ginecologia", "obstetrícia", "parto", "cesariana", e o período assumido para a busca das publicações iniciava-se na primeira metade do século XX. A associação entre "busca avançada" agregando "assunto" e "local de publicação: brasil", assim como "idioma: português", quando não havia a opção de busca por local de publicação, foi realizada para auxiliar a delimitação do levantamento de acordo com o interesse do grupo de pesquisa em questão. A seleção do tipo de publicação em "periódicos", "revistas", ou "publicações seriadas" buscou contemplar as possibilidades de termos que se julgou serem mais abrangentes no que se referia à produção de periódicos sobre a temática. Como exceção, a busca na base de dados da Biblioteca da Academia Nacional de Medicina, que não possui nenhum tipo de filtro de 
busca desses descritos acima, foi realizada apenas por assunto, com refinação individual da pesquisadora responsável, analisando os registros separadamente.

Nessa etapa foram localizados periódicos sobre ginecologia e obstetrícia em oito bibliotecas ou bases de dados, apresentadas nas Tabelas 1 e 2:

Tabela 1: Quantitativo geral de periódicos localizados

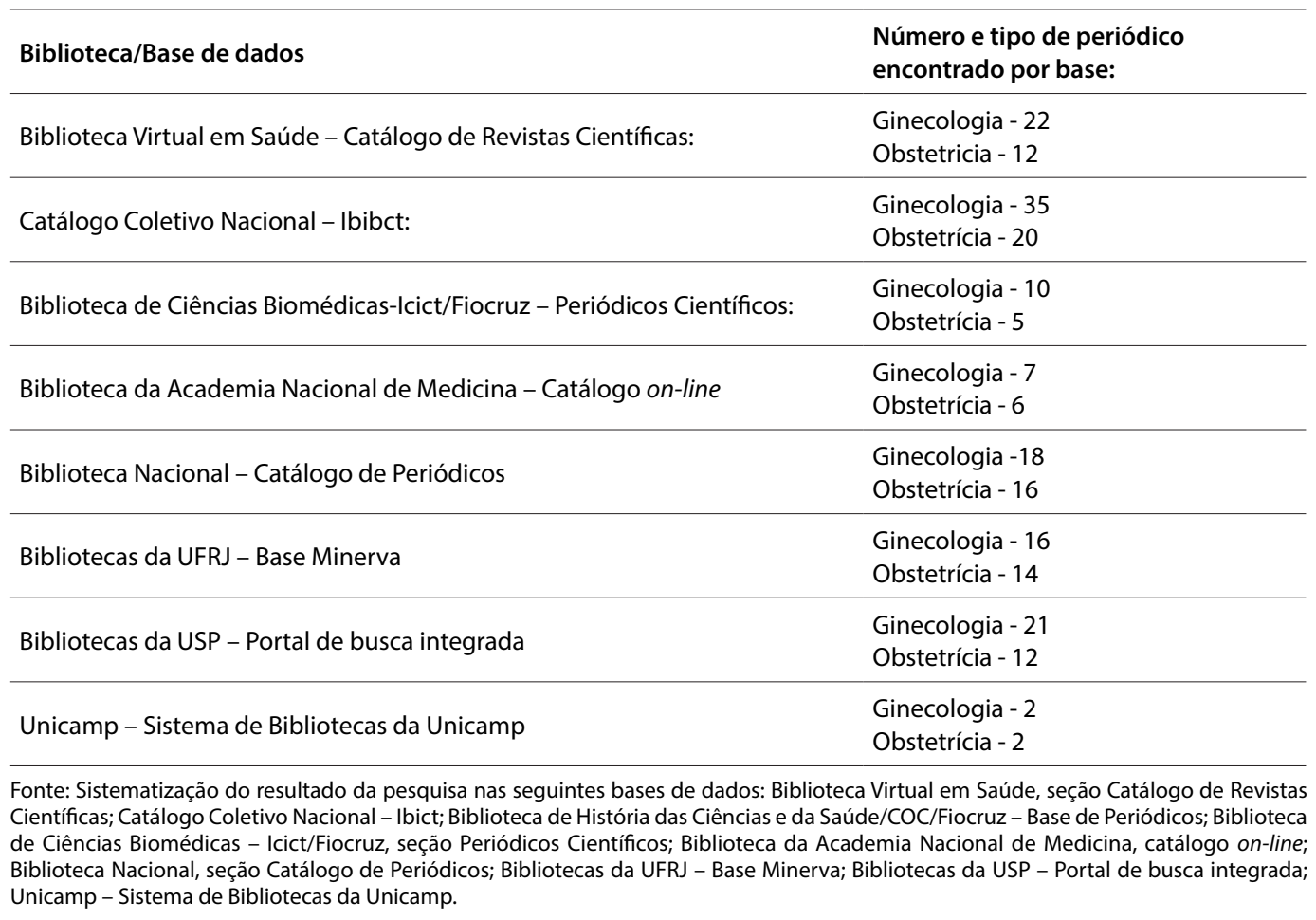

Tabela 2: Nome e período de publicação

\begin{tabular}{ll}
\hline Nome do periódico & Período de publicação \\
\hline Anais Brasileiros de Ginecologia & $1936-1969$ \\
\hline Jornal Brasileiro de Ginecologia/JBG: Jornal Brasileiro de Ginecologia & $1970-1999$ \\
\hline Anais da Clínica Ginecológica da Faculdade de Medicina da Universidade de São Paulo & $1947-1958$ \\
\hline Anais do Departamento de Obstetrícia e Ginecologia da Faculdade de Medicina da & $1959-1962$ \\
Universidade de São Paulo & $1949-1960$ \\
\hline Anais da Clínica Ginecológica da Santa Casa de São Paulo & $1955-1960$ \\
\hline Anais da Maternidade de São Paulo & $1968-1970$ \\
\hline Anais Nordestinos de Ginecologia e Obstetrícia & $1971-1984 / s . i$ \\
\hline Ango: Anais Nordestinos de Ginecologia e Obstetrícia & $1946 / 1947-s . i$ \\
\hline Arquivos da Universidade da Bahia & $1961-1963 / s . i$ \\
\hline Arquivos de Obstetrícia e Ginecologia & $1958-1960$ \\
\hline Obstetrícia prática & $1960-1964$ \\
\hline Boletim do Centro de Estudos da Sociedade Acadêmica da Maternidade-Escola & {$[1977 ?]-1983 / s . i$} \\
\hline Boletim do DGO &
\end{tabular}


Tabela 2: Nome e período de publicação (cont.)

\begin{tabular}{|c|c|}
\hline Nome do periódico & Período de publicação \\
\hline Boletim do Instituto de Ginecologia da Universidade Federal do Rio de Janeiro & 1964-1966 \\
\hline Caderno de Ginecologia e Obstetrícia & s.i \\
\hline Clínica Obstétrica & $1928-1931$ \\
\hline Clínicas Obstétricas e Ginecológicas (edição em português) & 1979-1992/s.i \\
\hline Femina & $1973-2011^{*}$ \\
\hline Ginecologia (Recife) & 1946-1964 \\
\hline Ginecologia atual *É Suplemento de: Atualidades Médicas (São Paulo) & $1965-1975$ \\
\hline Ginecologia Brasileira & 1969-1977 \\
\hline Ginecologia e Obstetrícia Atual & 1992-2002/s.i \\
\hline Ginecologia e Obstetrícia Brasileiras & 1978-1988/s.i \\
\hline GO (São Paulo): Revista de Atualização em Ginecologia e Obstetrícia & 1967-1973 \\
\hline Medicina Hospitalar (São Paulo) & $1974-1975$ \\
\hline Gynaecia atualização continuada em Ginecologia \& Obstetrícia & 1997/s.i \\
\hline Jornal da Febrasgo / Federação Brasileira das Sociedades de Ginecologia e Obstetrícia & 1994-2012/s.i \\
\hline Maternidade e Infância (São Paulo) & $1945-1976$ \\
\hline RBGO: Revista Brasileira de Ginecologia e Obstetrícia & 1986-2009* \\
\hline Revista Brasileira de Ginecologia \& Obstetrícia & 1979-1984 \\
\hline $\begin{array}{l}\text { Revista Brasileira de Medicina - Ginecologia e Obstetrícia/RBM - Ginecologia e Obstetrícia; } \\
\text { 2. Revista de Atualização em Ginecologia e Obstetrícia }\end{array}$ & 1989-1998/s.i \\
\hline Revista Brasileira de Saúde Materno Infantil/Rev. Bras. Saúde Mater. Infant. (on-line), 1806-9304 & $2001-2010^{*}$ \\
\hline Revista Científica do Hospital Materno Infantil Presidente Vargas & 1980-1983 \\
\hline Revista Científica/Revista Cientifica: Maternidade, Infância e Ginecologia & 1985-1998/s.i \\
\hline Revista da maternidade A Casa da Mãe Pobre & $1939-1940$ \\
\hline Revista da Maternidade-Escola Assis Chateaubriand & 1994-1995/s.i \\
\hline Revista da Sogia-BR & $2008-2010^{* *}$ \\
\hline Revista de Ginecologia e Obstetrícia (1907) & $1907-1978$ \\
\hline Revista de Ginecologia e Obstetrícia (1990) & 1990-2004/s.i \\
\hline Revista de Obstetrícia e Ginecologia de São Paulo & 1935/1936-1946/1947 \\
\hline Revista de Tocoginecologia & 1968-1973/s.i \\
\hline Revista do Imip/Revista do Instituto Materno Infantil de Pernambuco & $1987-2000$ \\
\hline Revista Ginecológica Brasileira & 1887-s.i \\
\hline Revista Obstétrica & 1861-s.i \\
\hline Revista Pernambucana de Maternidade e Infância & 1948-1951 \\
\hline Sinopse de Ginecologia e Obstetrícia & 1973-2002/s.i \\
\hline Temas de Ginecologia, Obstetrícia, Neonatologia e Pediatria & 1974-s.i \\
\hline $\begin{array}{l}\text { * Revistas ainda em publicação, data final identificada referente ao último número encontrado nas bases } p \\
\text { ** Última edição disponível em 2015, on-line. } \\
\text { s.i: Sem identificação de encerramento da publicação. }\end{array}$ & esquisadas. \\
\hline \multicolumn{2}{|c|}{$\begin{array}{l}\text { Fonte: Sistematização do resultado da pesquisa nas seguintes bases de dados: Biblioteca Virtual em Saúde, seção Catálogo de Revistas } \\
\text { Científicas; Catálogo Coletivo Nacional - Ibict; Biblioteca de História das Ciências e da Saúde/COC/Fiocruz - Base de Periódicos; Biblioteca } \\
\text { de Ciências Biomédicas - Icict/Fiocruz, seção Periódicos Científicos; Biblioteca da Academia Nacional de Medicina, catálogo on-line; } \\
\text { Biblioteca Nacional, seção Catálogo de Periódicos; Bibliotecas da UFRJ - Base Minerva; Bibliotecas da USP - Portal de busca integrada; } \\
\text { Unicamp - Sistema de Bibliotecas da Unicamp. }\end{array}$} \\
\hline
\end{tabular}


A Tabela 2 é referente ao demonstrativo dos títulos e dos períodos de publicação, contemplando o ano inicial e final do periódico, considerando a existência de lacunas referentes ao possível encerramento de algumas publicações. A pesquisa definiu como período de estudo o século XX, incorporando, porém, dois periódicos do final do século XIX, identificados no levantamento de fontes, o que pode significar o início do desenvolvimento de uma produção especializada na área. Foi decidido mantê-los na listagem como referência para pesquisas futuras, sem, no entanto, aprofundar a análise sobre o período em questão devido à delimitação temporal da presente pesquisa.

No caso das revistas em que houve uma alteração do título original, identificadas na Tabela 2, optamos, para este estudo, por manter a data inicial e final dos dois títulos, assim como as duas identidades editoriais que marcaram a história da publicação em momentos distintos, não nos detendo, contudo, nas causas das referidas mudanças.

A duração dos periódicos vai de um a setenta anos, e suas origens variam entre universidades, sociedades médicas, editoras e versões on-line. Houve um maior número de publicações iniciadas entre as décadas de 1960 e 1980 e uma maior concentração, considerando o total de revistas, no Sudeste, sendo o estado de São Paulo o que apresenta o maior número de periódicos. Os outros estados que aparecem são Rio de Janeiro, como o segundo local de maior produção, seguido de Recife, Rio Grande do Sul, Ceará, Santa Catarina, Bahia e Paraíba (Tabela 3).

Tabela 3: Referência editorial

\begin{tabular}{|c|c|}
\hline Nome do periódico & Localização de origem/editora \\
\hline \multicolumn{2}{|l|}{ Anais Brasileiros de Ginecologia } \\
\hline Jornal Brasileiro de Ginecologia & $\begin{array}{l}\text { ISSN 0368-1416. Menção de Responsabilidade: Centro } \\
\text { de Estudos Maternidade-Escola do Rio de Janeiro, } \\
\text { Universidade }\end{array}$ \\
\hline $\begin{array}{l}\text { Anais da Clínica Ginecológica da Faculdade de Medicina } \\
\text { da Universidade de São Paulo }\end{array}$ & $\begin{array}{l}\text { Universidade de São Paulo, Faculdade de Medicina, } \\
\text { Clínica Ginecológica (SP) }\end{array}$ \\
\hline $\begin{array}{l}\text { Anais do Departamento de Obstetrícia e Ginecologia da } \\
\text { Faculdade de Medicina da Universidade de São Paulo }\end{array}$ & $\begin{array}{l}\text { Universidade de São Paulo, Faculdade de Medicina, } \\
\text { Departamento de Obstetrícia e Ginecologia (SP) }\end{array}$ \\
\hline Anais da Clínica Ginecológica da Santa Casa de São Paulo & Santa Casa de São Paulo (SP) \\
\hline Anais da Maternidade de São Paulo & Associação Maternidade de São Paulo (SP) \\
\hline Anais Nordestinos de Ginecologia e Obstetrícia & Sociedade de Ginecologia e Obstetrícia \\
\hline Ango: Anais Nordestinos de Ginecologia e Obstetrícia & Sociedade de Ginecologia e Obstetrícia \\
\hline Arquivos da Universidade da Bahia & Universidade da Bahia, Faculdade de Medicina (BA) \\
\hline Arquivos de Obstetrícia e Ginecologia & Clínica Infantil do Ipiranga. Centro de Estudos \\
\hline Obstetrícia Prática & Clínica Infantil do Ipiranga. Centro de Estudos \\
\hline $\begin{array}{l}\text { Boletim do Centro de Estudos da Sociedade Acadêmica } \\
\text { da Maternidade-Escola }\end{array}$ & Universidade do Brasil. Maternidade-Escola (RJ) \\
\hline Boletim do DGO & $\begin{array}{l}\text { Associação Paulista de Medicina. Departamento de } \\
\text { Ginecologia e Obstetrícia (SP) }\end{array}$ \\
\hline $\begin{array}{l}\text { Boletim do Instituto de Ginecologia da Universidade } \\
\text { Federal do Rio de Janeiro }\end{array}$ & UFRJ. Instituto de Ginecologia (RJ) \\
\hline Caderno de Ginecologia e Obstetrícia & Grupo Editorial Moreira Jr. \\
\hline Clínica Obstétrica & $\mathrm{x}$ \\
\hline
\end{tabular}


Tabela 3: Referência editorial (cont.)

\begin{tabular}{|c|c|}
\hline Nome do periódico & Localização de origem/editora \\
\hline $\begin{array}{l}\text { Clínicas Obstétricas e Ginecológicas (edição em } \\
\text { português) }\end{array}$ & Interamericana \\
\hline Femina & ISSN 0100-7254 \\
\hline Ginecologia (Recife) & ISSN 0103-8680 \\
\hline Ginecologia Atual & Expansão \\
\hline Ginecologia Brasileira & $\begin{array}{l}\text { ISSN0046-5941 Menção de Responsabilidade: Sociedade } \\
\text { Brasileira de Citologia }\end{array}$ \\
\hline Ginecologia e Obstetrícia Atual & Publicações Científicas ISSN 1414-3534 \\
\hline Ginecologia e Obstetrícia Brasileiras & $\begin{array}{l}\text { ISSN0103-2771 Menção de Responsabilidade } \\
\text { Universidade de São Paulo. Faculdade de Medicina, } \\
\text { Departamento de Obstetrícia e Ginecologia }\end{array}$ \\
\hline $\begin{array}{l}\text { GO (São Paulo): Revista de Atualização em Ginecologia e } \\
\text { Obstetrícia }\end{array}$ & ISSN 0016-3597 \\
\hline Medicina Hospitalar (São Paulo) & Serpel \\
\hline $\begin{array}{l}\text { Gynaecia: atualização continuada em ginecologia \& } \\
\text { obstetrícia }\end{array}$ & Grupo ARS Curandi Eleá Ciência \\
\hline $\begin{array}{l}\text { Jornal da Febrasgo/Federação Brasileira das Sociedades } \\
\text { de Ginecologia e Obstetrícia }\end{array}$ & $\begin{array}{l}\text { Federação Brasileira das Sociedades de Ginecologia e } \\
\text { Obstetrícia }\end{array}$ \\
\hline Maternidade e Infância (São Paulo) & $\begin{array}{l}\text { Editora: Legião Brasileira de Assistência; Arquivos Médico- } \\
\text { Sociais }\end{array}$ \\
\hline RBGO: Revista Brasileira de Ginecologia e Obstetrícia & $\begin{array}{l}\text { ISSN0100-7203 Menção de responsabilidade: Federação } \\
\text { Brasileira das Sociedades de Ginecologia e Obstetrícia }\end{array}$ \\
\hline Revista Brasileira de Ginecologia \& Obstetrícia & $\begin{array}{l}\text { ISSN 0100-7203 ISSN online 1806-9339. Menção de } \\
\text { Responsabilidade: Federação Brasileira das Sociedades } \\
\text { de Ginecologia e Obstetrícia }\end{array}$ \\
\hline Revista Brasileira de Medicina - Ginecologia e Obstetrícia & Moreira Jr. \\
\hline Revista Brasileira de Saúde Materno Infantil & $\begin{array}{l}\text { Menção de Responsabilidade: Instituto de Medicina } \\
\text { Integral Professor Fernando Figueira ISSN 1519-3829 ISSN } \\
\text { on-line 1806-9304 }\end{array}$ \\
\hline Revista Científica & $\begin{array}{l}\text { ISSN0102-5120 Menção de Responsabilidade: Centro de } \\
\text { Aperfeiçoamento das Equipes de Saúde, Hospital Infantil } \\
\text { Presidente Vargas }\end{array}$ \\
\hline $\begin{array}{l}\text { Revista Científica do Hospital Materno Infantil Presidente } \\
\text { Vargas }\end{array}$ & $\begin{array}{l}\text { Menção de responsabilidade: Hospital Materno Infantil } \\
\text { Presidente Vargas }\end{array}$ \\
\hline Revista da maternidade A Casa da Mãe Pobre & $\begin{array}{l}\text { Menção de Responsabilidade: Associação Maternidade } \\
\text { de São Paulo }\end{array}$ \\
\hline Revista da Maternidade-Escola Assis Chateaubriand & $\begin{array}{l}\text { Menção de responsabilidade: UFCE. Maternidade-Escola } \\
\text { Assis Chateaubriand }\end{array}$ \\
\hline Revista da Sogia-BR & $\begin{array}{l}\text { Menção de responsabilidade: Sociedade Brasileira de } \\
\text { Obstetrícia e Ginecologia da Infância e Adolescência }\end{array}$ \\
\hline Revista de Ginecologia e Obstetrícia (1907) & $\begin{array}{l}\text { ISSN0034-8287 Menção de Responsabilidade: Associação } \\
\text { Maternidade de São Paulo. Sociedade Paulista de } \\
\text { Perinatologia }\end{array}$ \\
\hline Revista de Ginecologia e Obstetrícia (1990) & ISSN0103-7714 Instituto da Mulher HC-FMUSP \\
\hline Revista de Obstetrícia e Ginecologia de São Paulo & $\mathrm{X}$ \\
\hline Revista de Tocoginecologia & $\begin{array}{l}\text { Menção de responsabilidade: Universidade da Paraíba, } \\
\text { Departamento de Obstetrícia e Ginecologia }\end{array}$ \\
\hline Revista do Imip & $\begin{array}{l}\text { ISSN0102-8847 Menção de Responsabilidade: Instituto } \\
\text { Materno Infantil de Pernambuco }\end{array}$ \\
\hline
\end{tabular}


Tabela 3: Referência editorial (cont.)

\begin{tabular}{ll}
\hline Nome do periódico & Localização de origem/editora \\
\hline Revista Ginecológica Brasileira & $\mathrm{X}$ \\
\hline Revista Obstétrica & $\mathrm{X}$ \\
\hline Revista Pernambucana de Maternidade e Infância & $\mathrm{X}$ \\
\hline Sinopse de Ginecologia e Obstetrícia & Moreira Jr. Editora Médica \\
\hline Temas de Ginecologia, Obstetrícia, Neonatologia e & $\mathrm{X}$ \\
\hline Pediatria &
\end{tabular}

Fonte: Sistematização do resultado da pesquisa nas seguintes bases de dados: Biblioteca Virtual em Saúde, seção Catálogo de Revistas Científicas; Catálogo Coletivo Nacional - Ibict; Biblioteca de História das Ciências e da Saúde/COC/Fiocruz - Base de Periódicos; Biblioteca de Ciências Biomédicas - Icict/Fiocruz, seção Periódicos Científicos; Biblioteca da Academia Nacional de Medicina, catálogo online; Biblioteca Nacional, seção Catálogo de Periódicos; Bibliotecas da UFRJ - Base Minerva; Bibliotecas da USP - Portal de busca integrada; Unicamp - Sistema de Bibliotecas da Unicamp.

A compreensão do contexto referente à concentração das revistas demanda análises específicas sobre cada periódico. No que diz respeito às publicações paulistas, por exemplo, há um estudo da pesquisadora Marcia Silva (2010), em artigo publicado sobre a produção de periódicos médicos e afins em São Paulo, com títulos referentes às áreas de ginecologia e obstetrícia, identificados pela autora em sua pesquisa, considerando também a primeira metade do século XX, que constam igualmente em nosso levantamento. ${ }^{1}$ No entanto, a autora identificou como continuidades, em seu estudo, os seguintes títulos: Revista de Ginecologia e Obstetrícia de São Paulo; Anais da Clínica Ginecológica; Anais do Departamento de Obstetrícia e Ginecologia; Ginecologia e Obstetrícia Brasileiras; Revista de Ginecologia e Obstetrícia, considerando-os uma única publicação.

O período da segunda metade do século XX, segundo Silva (2010), foi marcado por grandes transformações no âmbito da ciência nacional, inclusive nas políticas científicas, com a criação de organismos reguladores de pesquisas como o Conselho Nacional de Pesquisa (CNPq), atual Conselho Nacional de Desenvolvimento Científico e Tecnológico, em 1951, e a Fundação de Amparo à Pesquisa do Estado de São Paulo (Fapesp), em 1962, fomentando pesquisas e publicações no âmbito acadêmico/científico no Brasil. Essa interpretação justifica, em primeira análise, a maior publicação de revistas no início da segunda metade do século XX, na análise mais ampla realizada no presente estudo.

A localização física dos acervos dos periódicos encontrados indica uma predominância de bibliotecas de universidades como locais de armazenamento e disponibilização de tais fontes documentais (Tabela 4), sendo a revista Temas de Ginecologia, Obstetrícia, Neonatologia e Pediatria o único título que não teve sua localização física identificada.

Tabela 4: Referência de localização física do acervo

Nome do periódico

Anais Brasileiros de Ginecologia

Jornal Brasileiro de Ginecologia

\section{Acervo}

Uerj. Centro Biomédico. Bib. setorial (1936-1968); UFF. Fac. de Medicina, Bib. setorial (1936-1968); Biblioteca Maternidade-Escola (1936-1969)

ANM (1970-1992); Fiocruz. IFF (1970-1998); Inca. Vila Isabel (1970-1997); Inca. BT (1970-1997); UFF. Bib. Nutrição (19791997); UFF. FM (1970-1998); UFRJ. CCS (1970-1990); UFRJ. Bib. da Maternidade-Escola (1970-1999) 
Tabela 4: Referência de localização física do acervo (cont.)

\section{Nome do periódico}

Anais da Clínica Ginecológica da Faculdade de Medicina da Universidade de São Paulo

Anais do Departamento de Obstetrícia e Ginecologia da Faculdade de Medicina da Universidade de São Paulo

Anais da Clínica Ginecológica da Santa Casa de São Paulo

Anais da Maternidade de São Paulo

Anais Nordestinos de Ginecologia e Obstetrícia

Ango: Anais Nordestinos de Ginecologia e Obstetrícia

Arquivos da Universidade da Bahia

Arquivos de Obstetrícia e Ginecologia

Obstetrícia Prática

Boletim do Centro de Estudos da Sociedade Acadêmica da Maternidade-Escola

Boletim do DGO

Boletim do Instituto de Ginecologia da Universidade Federal do Rio de Janeiro

Caderno de Ginecologia e Obstetrícia

Clínica Obstétrica

Clínicas Obstétricas e Ginecológicas (edição em português)

Femina

Ginecologia (Recife)

Ginecologia Atual

Ginecologia Brasileira

Ginecologia e Obstetrícia Atual

Ginecologia e Obstetrícia Brasileiras

GO (São Paulo): Revista de Atualização em Ginecologia e Obstetrícia

Medicina Hospitalar (São Paulo)

Gynaecia atualização continuada em ginecologia \& obstetrícia

Jornal da Febrasgo/Federação Brasileira das Sociedades de Ginecologia e Obstetrícia

Maternidade e Infância (São Paulo)

\section{Acervo}

UFRJ. CCS. BC. (1947-1958); UFRJ. Bib. da MaternidadeEscola (1947-1951)

UFRJ. CCS. B.C. (1959-1962)

UFBA. Fac. de Med. Bib. (1947-1958); PUC de Campinas

(1954-1958); USP. Fac. de Med. Serviço de Bib. e Doc. (1947-1958)

UFRJ. CCS. B.C. (1956-1961); UFRJ. Bib. da MaternidadeEscola (1956-1961)

UFRJ. Bib. Da Maternidade-Escola (1971-1983); Inca. Vila Isabel (1971-1984)

UFRJ. Bib. Da Maternidade-Escola (1971-1983); Inca. Vila Isabel (1971-1984)

UFRJ. MN. BT (1948); USP. FM. SBD (1946-1949); Instituto Bras. de Invest. do Thórax BT(BA) (1946-1952)

ANM. BT (1962-1963); USP. FM. SBD (1961-1963)

USP. FM (1958-1961); Ufpe. CCS (1958-1961)

UFRJ. Maternidade-Escola

BN (1980-1983)

Fiocruz. Bib. de C. Biomédicas (1965-1966); UFRJ. CCS (1964-1966); UFRJ. Bib. da Maternidade-Escola (19641966)

USP. FSP; USP. FM de Ribeirão Preto

UFRJ. Bib. da Maternidade-Escola (1928-1931)

UFRJ. Bib. da Maternidade-Escola (1979-1992)

ANM (1974-1989); Fiocruz. IFF. BT (1975-2007); UFF (19732011); Inca. HLGL. Vila Isabel (1973-1989)

Uerj. Centro Biomédico. Bib. Set. (1946-1959); UFRJ. CCS (1949-1963); UFRJ. Bib. da Maternidade-Escola (19491964)

Fiocruz. Bib. de C. Biomédicas (1972-1974); USP. Fac. de Odontol. de Bauru (1967-1978)

ANM (1967-1977); Uerj. C. Biomédico (1969-1977); UFF. FM (1969-1977); UFRJ. CCS (1969-1977); UFRJ. Bib. da Maternidade-Escola (1969-1977)

UFF. FM. BT (1993-2001); UFRJ. Bib. da MaternidadeEscola (1992-2002)

UFRJ. Bib. da Maternidade-Escola (1978-1988)

ANM (1967-1973); Uerj. C. Biomédico. Bib. Set. (1967-

1973); UFF. FM. Bt (1967-1973); UFRJ. CCS (1967-1973);

UFRJ. Bib. da Maternidade-Escola (1967-1973)

UFF. FM. BT (1974); USP. FM (1974-1975)

USP. Fac. Med.

Biblioteca Nacional (1994-2012)

ANM (1952-1976); Uerj. C. B. Bib. Set. (1950-1976); UFF. BNO (1961-76); UFF. FM. BT (1945-76); UFRJ. CCS. BC (1947-1975) 
Tabela 4: Referência de localização física do acervo (cont.)

\begin{tabular}{|c|c|}
\hline Nome do periódico & Acervo \\
\hline RBGO: Revista Brasileira de Ginecologia e Obstetrícia & $\begin{array}{l}\text { ANM (1985-1989); Inca. BT (1981-1993); Inca. HLGL. Vila } \\
\text { Isabel (1979-1989); UFRJ. CCS (1979-2009); UFRJ. Bib. da } \\
\text { Maternidade-Escola }\end{array}$ \\
\hline Revista Brasileira de Ginecologia \& Obstetrícia & $\begin{array}{l}\text { ANM (1985-1989); Inca. HLGL. Vila Isabel (1979-1989); } \\
\text { Inca. BT (1981-1993); UFF. FM (1979-2011); UFRJ. CCS } \\
\text { (1979-2009) }\end{array}$ \\
\hline Revista Brasileira de Medicina - Ginecologia e Obstetrícia & $\begin{array}{l}\text { UFF. FM (1991-1998); UFRJ. Bib. da Maternidade-Escola } \\
(1989-1998)\end{array}$ \\
\hline Revista Brasileira de Saúde Materno Infantil & Fiocruz. IFF (2001-2007); UFF. FM (2001-2010) \\
\hline Revista Científica & $\begin{array}{l}\text { PUC. RS. BC. Bib. de Medicina (1985-1996); Bireme. BT (SP) } \\
(1985-1998)\end{array}$ \\
\hline $\begin{array}{l}\text { Revista Científica do Hospital Materno Infantil Presidente } \\
\text { Vargas }\end{array}$ & $\begin{array}{l}\text { UFRJ. Bib. da Maternidade-Escola (1981); PUC. RS. BC } \\
(1980-83)\end{array}$ \\
\hline Revista da Maternidade A Casa da Mãe Pobre & USP. FM (1939-1940) \\
\hline Revista da Maternidade-Escola Assis Chateaubriand & PUC. Campinas (1994-95); UNB. BC (1994-1995) \\
\hline Revista da Sogia-BR & Biblioteca Nacional (2006-2010) \\
\hline Revista de Ginecologia e Obstetrícia (1907) & $\begin{array}{l}\text { ANM (1922-1976); Uerj (1907-1977); UFF. FM (1922-1978); } \\
\text { UFRJ. CCS (1910-1977); UFRJ. Bib. da Maternidade-Escola } \\
\text { (1915-1978) }\end{array}$ \\
\hline Revista de Ginecologia e Obstetrícia (1990) & $\begin{array}{l}\text { UFF. FM (1990-2004); UFRJ. Bib. da Maternidade-Escola } \\
(1990-2004)\end{array}$ \\
\hline Revista de Obstetrícia e Ginecologia de São Paulo & $\begin{array}{l}\text { UFRJ. CCS (1936-1947); UFRJ. Bib. da Maternidade-Escola } \\
(1935-1947)\end{array}$ \\
\hline Revista de Tocoginecologia & $\begin{array}{l}\text { UFRJ. Bib. da Maternidade-Escola (1968-1971); UFPB. BC } \\
(1968-1973)\end{array}$ \\
\hline Revista do IMIP & UFF. FM. BT (1999-2000); Bireme. BT (1987-2000) \\
\hline Revista Ginecológica Brasileira & UFRJ. CCS. BC (1887-v.1) \\
\hline Revista Obstétrica & UFRJ. CCS (1861) \\
\hline Revista Pernambucana de Maternidade e Infância & UFRJ. CCS (1949-1951); Ufba. FM. BT (1948-1951) \\
\hline Sinopse de Ginecologia e Obstetrícia & $\begin{array}{l}\text { UFF. FM. BT (1973-1983); UFRJ. Bib. da Maternidade- } \\
\text { Escola (1995-2002) }\end{array}$ \\
\hline $\begin{array}{l}\text { Temas de Ginecologia, Obstetrícia, Neonatologia e } \\
\text { Pediatria }\end{array}$ & Não encontrada \\
\hline
\end{tabular}

Fonte: Sistematização do resultado da pesquisa nas seguintes bases de dados: Biblioteca Virtual em Saúde, seção Catálogo de Revistas Científicas; Catálogo Coletivo Nacional - Ibict; Biblioteca de História das Ciências e da Saúde/COC/Fiocruz - Base de Periódicos; Biblioteca de Ciências Biomédicas - Icict/Fiocruz, seção Periódicos Científicos; Biblioteca da Academia Nacional de Medicina, catálogo on-line; Biblioteca Nacional, seção Catálogo de Periódicos; Bibliotecas da UFRJ - Base Minerva; Bibliotecas da USP - Portal de busca integrada; Unicamp - Sistema de Bibliotecas da Unicamp.

Após o levantamento inicial, as buscas foram direcionadas para os acervos localizados no Rio de Janeiro, para a realização da análise dos periódicos encontrados. O acervo da biblioteca da Maternidade-Escola da Universidade Federal do Rio de Janeiro foi o local com mais periódicos encontrados nas áreas de ginecologia e obstetrícia para o período pesquisado, sendo, por isso, escolhido como o primeiro local para a realização da análise de acervo.

O ensino obstétrico no Brasil desenvolveu-se a partir do início do século XIX, na Escola Anatômica, Cirúrgica e Médica do Rio de Janeiro e na Escola de Cirurgia da Bahia, 
posteriormente denominadas Academias Médico-Cirúrgicas. A cadeira de parto foi integrada em definitivo quando essas academias se transformaram nas faculdades de medicina desses estados, criadas em 1832 (Rohden, 2009). Inaugurada em 1904 como Maternidade-Escola do Rio de Janeiro, localizada no bairro de Laranjeiras, essa instituição foi incorporada à Faculdade de Medicina em 1918, tornando-se Maternidade-Escola do Rio de Janeiro, ampliando as opções de atendimento e ensino obstétrico no Rio de Janeiro, antes restrito à Santa Casa de Misericórdia. De acordo com Ana Paula Vosne Martins (2004, p.204), a maternidade foi conhecida na época "como um exemplo de organização e de aplicação dos preceitos científicos da obstetrícia e da ginecologia", além do destaque como local de ensino, principalmente após a inserção do doutor Fernando Magalhães como professor substituto na cadeira de clínica obstétrica.

O acervo da Biblioteca da Maternidade-Escola da UFRJ está organizado por coleções, no segundo andar da biblioteca, sem muito acesso dos usuários devido à especificidade da formação na instituição demandar um uso maior das bibliografias atualizadas sobre o campo. Nessa biblioteca foram encontrados os seguintes títulos de periódicos: Anais da Clínica Ginecológica da Faculdade de Medicina da Universidade de São Paulo; Anais da Maternidade de São Paulo; Arquivos de Clínica Ginecológica; Boletim do Centro de Estudos da Sociedade Acadêmica da Maternidade-Escola; Clínica Obstétrica; Fundação Clara Basbaum; Boletim do Centro de Estudos; Ginecologia (Recife); Maternidade e Infância (São Paulo); Revista de Obstetrícia e Ginecologia de São Paulo; Revista Brasileira de Ginecologia e D’Obstetrícia e Anais Brasileiros de Ginecologia.

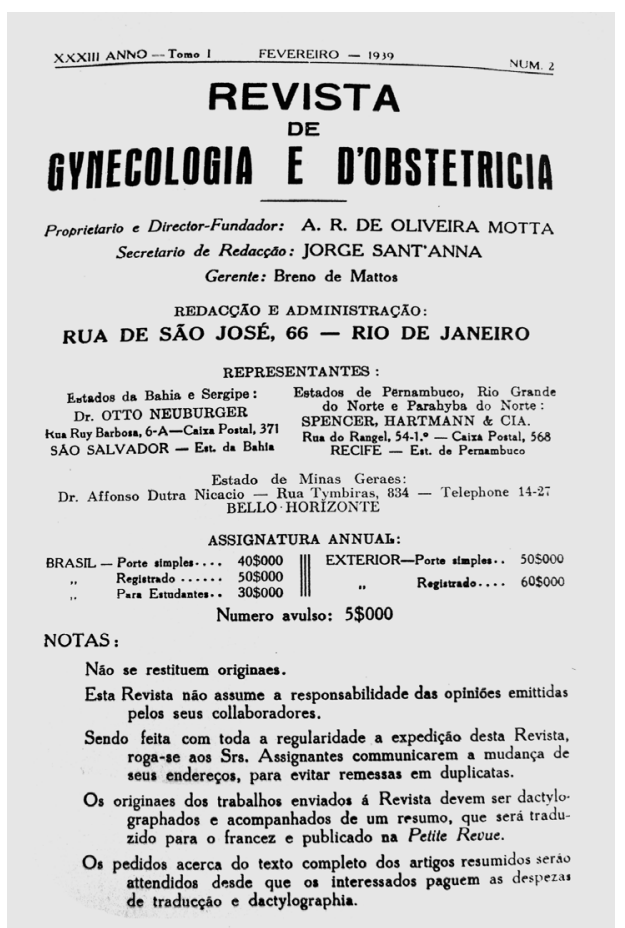

Figura 1: Sumário da Revista de Ginecologia e D'Obstetrícia, ano 33, t.1, n.1, jan. 1939

(Fonte: Acervo da Biblioteca da Maternidade-Escola da Universidade Federal do Rio de Janeiro) 


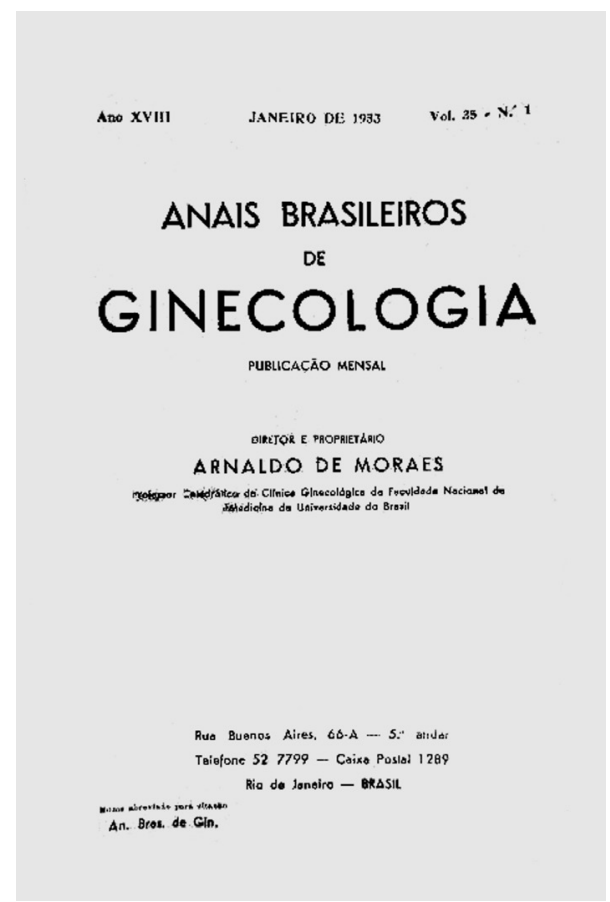

Figura 2: Capa dos Anais Brasileiros de Ginecologia, ano 18, v.35, n.1, jan. 1933 (Fonte: Acervo da Biblioteca da Maternidade-Escola da Universidade Federal do Rio de Janeiro)

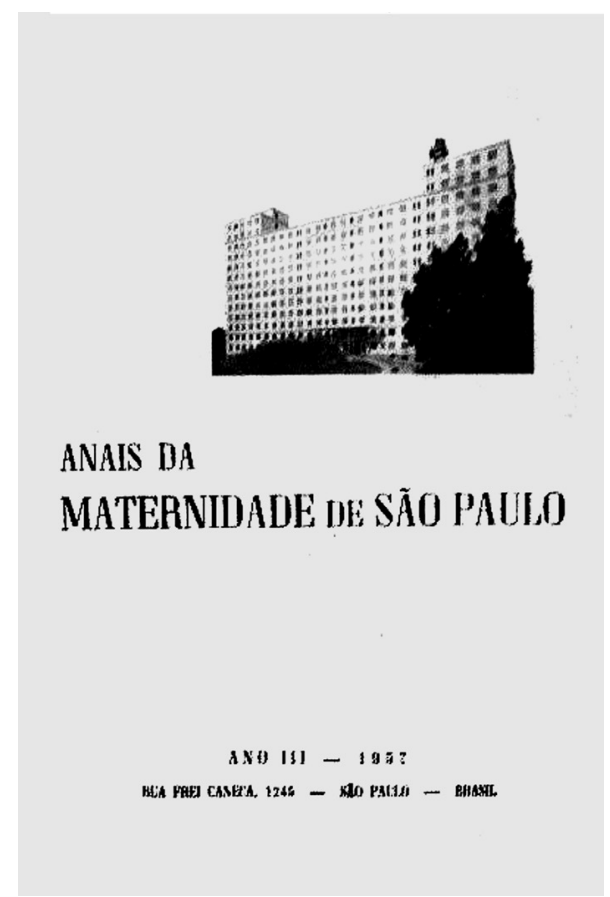

Figura 3: Capa dos Anais da Maternidade de São Paulo, ano 3, 1957 (Fonte: Biblioteca da Maternidade-Escola da Universidade Federal do Rio de Janeiro) 
Diversas temáticas referentes ao ensino da obstetrícia, suas técnicas e procedimentos utilizados no parto e na cesárea, junto a relatos de cirurgias ginecológicas, são abordadas nas revistas encontradas. Casos de câncer e outras patologias referentes ao aparelho reprodutivo feminino, além de condições obstétricas, assim como a discussão de casos clínicos específicos, também são abordados nesses periódicos. A influência de temáticas discutidas em âmbito internacional relacionadas às áreas de ginecologia e obstetrícia, como a abordagem do parto sem dor, aparece em forma de artigos e notas, contribuindo para a compreensão da concepção sobre o tema no Brasil em diferentes momentos. Alguns artigos possuem ilustrações e/ou fotografias dos casos clínicos estudados, relatórios quantitativos de atendimento em maternidades, entre outras informações de grande valor para a análise histórica da construção do conhecimento científico na área, assim como da assistência à saúde da mulher no Brasil.

A título de exemplo sobre a amplitude dos assuntos abordados, destacamos, no número de agosto de 1939 da Revista de Ginecologia e D'Obstetrícia, o artigo escrito por um médico atuante no estado do Paraná, doutor Victor do Amaral. Nesse estudo é observada a descrição da prática definida pelo autor como "parto secreto", que consistia na assistência às mulheres gestantes cujos fetos eram considerados "ilegítimos" ou fruto de "defloramento". Nesse contexto, o médico descreve a negativa na prática do aborto com a oferta de assistência ao parto de forma secreta, mencionando também a presença de parteira dentro da maternidade. Artigos como esse constituem fontes importantes para os estudos que investigam, entre outros temas, o discurso médico sobre aborto, as condições morais vigentes na sociedade que influenciavam a prática assistencial e ainda o convívio entre o profissional médico e a parteira dentro de um espaço institucionalizado de assistência.

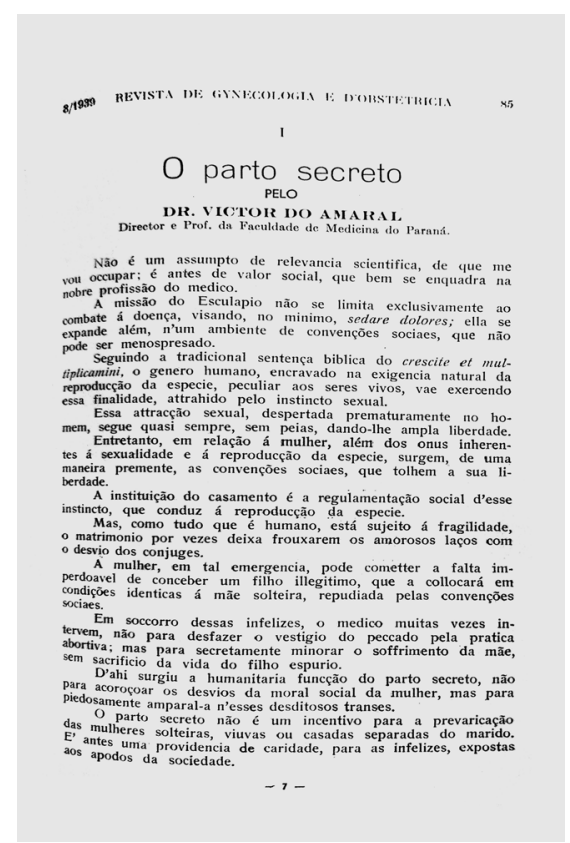

Figura 4: Primeira página do artigo "O parto secreto", Revista de Ginecologia e D’Obstetrícia, ago. 1939 (Fonte: Acervo da Biblioteca da Maternidade-Escola da Universidade Federal do Rio de Janeiro) 
Em outro periódico localizado na biblioteca da Maternidade-Escola, a saber, a revista Clínica Obstétrica, destacamos o artigo intitulado "A orientação obstétrica no Brasil: ensino e pesquisa". Trata-se de um texto apresentado em uma conferência realizada em Paris, assinado pelo médico Fernando Magalhães, em junho de 1930. O periódico apresenta-se como uma publicação destinada ao registro dos trabalhos desse profissional, conforme exposto na capa da publicação. No artigo mencionado, o autor aborda questões relevantes para a compreensão histórica da atuação dos médicos e, especificamente, da constituição da prática obstétrica na sociedade. Fernando Magalhães destaca a construção de uma nova maternidade no Rio de Janeiro, citando a importância de tratar-se de uma instituição separada de um hospital. Reafirma a visão do papel social específico da mulher na sociedade naquele contexto, definido pelo seu sexo biológico e, atrelado a ele, o papel da obstetrícia como mantenedora das "obrigações raciais". Assim, segundo Magalhães (1930, p.74),

pelo sexo, a mulher liga-se à eternidade da espécie; o aparelho genital feminino não é propriedade individual, é a propriedade da raça e a mulher é dele depositário. A obstetrícia prepara a mulher na compreensão deste grande papel que implica estrutura física e compleição moral e apela para os responsáveis, legisladores e condutores de opinião no dever de evitar, com a obra de proteção integral do sexo, o esquecimento dos atributos e das obrigações femininas.

A defesa de um espaço hospitalar reservado às gestantes e aos recém-nascidos consistia em uma defesa dos médicos e de alguns segmentos urbanos, na virada do século XIX para o XX, preocupados com a alta taxa de mortalidade infantil, que não correspondia ao ideal de construção da nacionalidade em voga (Barreto, 2011). O trecho do texto de Magalhães apresentado acima vai ao encontro da abordagem de Fabiola Rohden (2009) na afirmação da construção de uma ciência da mulher baseada na diferença entre os sexos e na maternidade como função social estabelecida para a mulher (Freire, 2009) no contexto higienista e nacionalista do início do século XX.

\section{Considerações finais}

Esta breve exposição do levantamento de periódicos de ginecologia e obstetrícia publicados no Brasil na primeira metade do século XX teve como objetivo principal disponibilizar o resultado de um estudo cujo foco encontra-se na análise histórica do processo de medicalização do corpo feminino e, mais especificamente, de medicalização do parto e do nascimento no Brasil, identificados nas revistas especializadas em ginecologia e obstetrícia.

A análise dos acervos com esse objetivo inicial, entretanto, revelou-nos a riqueza de temas diferenciados nas revistas encontradas tanto para os estudos históricos sobre a construção da obstetrícia e ginecologia como campo no Brasil quanto para o estudo da trajetória de técnicas e abordagens médicas desenvolvidas nestas áreas da medicina, além das questões políticas e sociais que as envolviam no momento de sua produção e divulgação, entre tantas outras possibilidades de análise. 


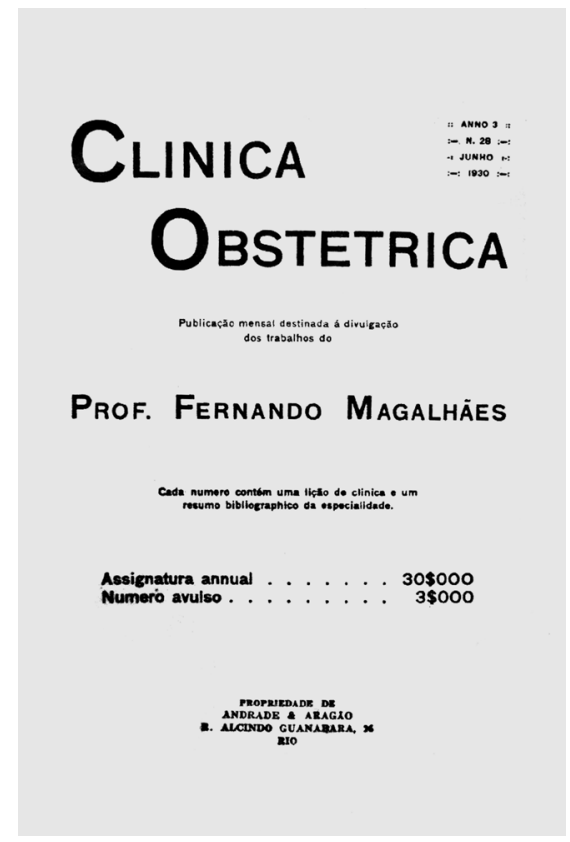

Figura 5: Capa da revista Clínica Obstétrica, ano 3, n.28, jun. 1930

(Fonte: Acervo da Biblioteca da Maternidade-Escola da Universidade Federal do Rio de Janeiro)

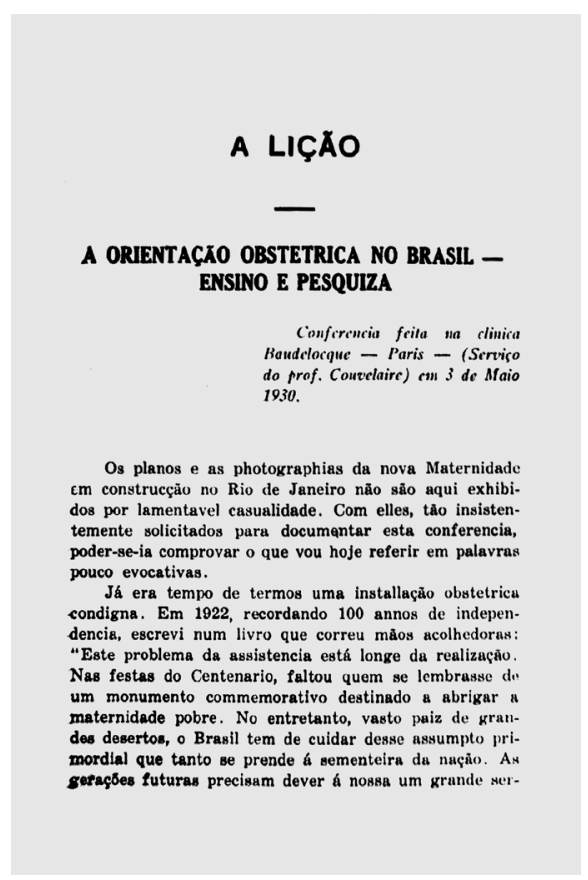

Figura 6: Primeira página do artigo “A orientação obstétrica no Brasil - ensino e pesquisa”, Clínica Obstétrica, ano 3, n.28, jun. 1930 (Fonte: Acervo da Biblioteca da Maternidade-Escola da Universidade Federal do Rio de Janeiro) 
Procuramos, dessa forma, apresentar o levantamento realizado em bibliotecas e demais instituições responsáveis pela guarda de acervos de periódicos no país, mais detidamente no Rio de Janeiro, e destacar o papel da publicação científica especializada nas áreas de ginecologia e obstetrícia no Brasil, buscando contribuir para a divulgação do conjunto de fontes que possam vir a subsidiar a reflexão histórica acerca desses campos disciplinares.

\section{NOTA}

${ }^{1}$ As revistas identificadas pela autora em sua pesquisa foram: Revista de Ginecologia e de Obstetrícia, do Centro de Estudos da Associação Maternidade de São Paulo; Revista de Obstetrícia e Ginecologia de São Paulo; Maternidade e Infância; Revista de Ginecologia e Obstetrícia de São Paulo; Anais da Clínica Ginecológica; Anais do Departamento de Obstetrícia e Ginecologia; Ginecologia e Obstetrícia Brasileiras; Revista de Ginecologia e Obstetrícia; Anais da Clínica Ginecológica da Santa Casa de São Paulo (Silva, 2010).

\section{REFERÊNCIAS}

BARRETO, Maria Renilda Nery.

Pro Matre: arquivo e fontes para a história da maternidade no Rio de Janeiro. História, Ciências, Saúde-Manguinhos, v.18, supl.1, p.295-301. 2011.

BONAN, Claudia; TEIXEIRA, Luiz Antonio; NAKANO, Andreza Rodrigues.

Absorção e metabolização dos hormônios sexuais e sua transformação em tecnologias contraceptivas: percursos do pensamento médico no Brasil. Ciência \& Saúde Coletiva, v.22, n.1, p.107-116. 2017.

FERREIRA, Luiz Otávio.

Os periódicos médicos e a invenção de uma agenda sanitária para o Brasil (1827-43). História, Ciências, Saúde-Manguinhos, v.6, n.2, p.331-351. 1999.

FREIRE, Maria Martha de Luna.

Mulheres, mães e médicos: discurso maternalista no Brasil. Rio de Janeiro: FGV. 2009.

FREITAS, Maria Helena.

Considerações acerca dos primeiros periódicos científicos brasileiros. Ciência da Informação, v.35, n.3, p.54-66. set.-dez. 2006.

FREITAS, Patrícia de.

Panorama da imprensa médica no Brasil: o lançamento da Revista de Ginecologia e D’Obstetrícia em 1907. Patrimônio e Memória, v.4, n.1, p.25-41. 2008.

FREITAS, Patrícia de.

Corpos de mulheres em (re)vista: a representação da menopausa na Revista de Ginecologia e d' Obstetrícia 1907-1978. Tese (Doutorado) - Universidade Federal de Santa Catarina, Florianópolis. 2005.

LUCA, Tania Regina de.

História dos, nos e por meio dos periódicos. In:
Pinsky, Carla Bassanesi (Org.). Fontes históricas. São Paulo: Contexto. p.111-154. 2008.

MAGALHÃES, Fernando.

A orientação obstétrica no Brasil: ensino e pesquisa. Revista Clínica Obstétrica, ano 3, n.28, p.69-86. 1930.

MARTINS, Ana Paula Vosne.

Visões do feminino: a medicina da mulher nos séculos XIX e XX. Rio de Janeiro: Editora Fiocruz. 2004.

MENEZES, Valderiza Almeida.

"Fiquei homem": maternidade, conhecimento e contracepção entre mulheres pobres de Fortaleza (1960-1980). Dissertação (Mestrado em História Social) - Universidade Federal do Ceará, Fortaleza. 2012.

MOTT, Maria Lúcia.

Assistência ao parto: do domicílio ao hospital (1830-1960). Projeto História, n.25, p.197-219. 2002.

ROHDEN, Fabíola.

Uma ciência da diferença: sexo e gênero na medicina da mulher. Rio de Janeiro: Editora Fiocruz. 2009.

SÁ, Dominichi Miranda de.

A ciência como profissão: médicos, bacharéis e cientistas no Brasil (1895-1935). Rio de Janeiro: Editora Fiocruz. 2006.

SILVA, Maria Regina Barros da. Periódicos médicos em São Paulo entre 1889 e 1950. In: Monteiro, Yara Nogueira. História da Saúde: olhares e veredas. São Paulo: Instituto de Saúde. 2010.

VIEIRA, Elizabeth Meloni.

A medicalização do corpo feminino. Rio de Janeiro: Editora Fiocruz. 2002. 